\title{
Shear properties evaluation of natural fibre reinforced epoxy composites using V-notch shear test
}

\author{
Jauhar Fajrin ${ }^{1, *}$ and Nasmi Herlina Sari ${ }^{2}$ \\ ${ }^{1}$ Civil Engineering Department, University of Mataram, 83126, Mataram, Indonesia \\ ${ }^{2}$ Mechanical Engineering Department, University of Mataram, 83126, Mataram, Indonesia
}

\begin{abstract}
This paper discusses the results of an experimental investigation on the shear properties of natural fibre composites using the V-Notch or Iosipescu shear test. The natural fiber composites were made of jute and hemp fibers reinforced epoxy polymer resin. The testing was conducted using an MTS machine equipped with a $10 \mathrm{kN}$ load cell in accordance with the ASTM standard D5379M. The testing machine was set-up to apply a load with a speed of $2 \mathrm{~mm} / \mathrm{min}$. It was found that the jute fiber composites (JFC) have an average shear stress of $25.56 \mathrm{MPa}$, while the hemp fiber composites (HFC) have an average value of $22.46 \mathrm{MPa}$. The loaddisplacement graphs showed a nearly linear trend at the initial loading stage that slightly deflected prior to reaching the maximum load. Most of the specimens in both groups, JFC and HFC, showed fractures in a diagonal cracking pattern, known as an off-axial failure mode. Only a few specimens collapsed across the notched section. Both are customary failure modes for this type of shear test.
\end{abstract}

Keywords: Shear properties, V-notch test, natural fiber, composites

\section{Introduction}

Natural fiber composites (NFC) are one of the most emerging natural-based products that recently have attracted more attention. As the name implies, NFC is a class of composite that contains natural fibres mixed with synthetic or bio-resins that are inherently environmentally beneficial. Other advantages of NFC are well documented in literatures, Suddel and Rosemaund [1] highlighted some advantages of NFC include low density, low cost, high toughness, acceptable specific strength properties and excellent acoustic properties thereby reducing noise. More recently, Sari et al. [2] developed a composite based on corn husk fiber that has superior acoustical properties. Initially, this emerging material was extensively investigated in relation to mechanical engineering applications, but it now has extended to other engineering fields such as in civil engineering. Many studies have also been focused on developing building components, among these was the hybrid structures for structural applications as reported by Fajrin [3] and Fajrin et al. [4-6].

\footnotetext{
*Corresponding author: jauhar.fajrin@unram.ac.id
} 
Natural fiber composites are particularly valued for their tensile and flexural properties [7], while shear and compressive properties are often ignored at the early stages of fiber composite development. Almeida Jr et al. [8] stated that only a few papers in the literature of fiber composite were linked to shear tests. One of the reason is probably due to the complexity of running the test, ensuring the presence of pure shear within the specimen. Broughton [9] stated that a shear test method should provide a region of pure shear stresses in the specimen throughout the linear or non-linear response regime. Shear properties must be evaluated particularly when the product is designed to carry a shear load. A comparatively low shear or compressive strength of a fiber composite reduces their promising application although it possesses high tensile or flexural properties [10].

One of the most commonly employed methods for shear testing is Iosipescu shear test, which is also known as the V-notched shear test [8]. As the name implied, the test was originally developed by Iosipescu [11]. Among the available shear test methods, the Iosipescu test has been widely used due to its ability to propagate an almost pure shear stress state within the notched section of the specimen [12]. As described in ASTM standard D5379M [13], the Iosipescu or V-notch uses a rectangular beam with a symmetrical centrally located v-notch. The beam is loaded by a special fixture, applying a shear loading at the vnotch. The specimen is inserted into the fixture with the notch located along the line of action of loading by means of an alignment that references the fixture. The two halves of the fixture are compressed by a testing machine, while the load is monitored.

Until now, there are only a few reported literatures dealing with the characterization of fiber composites using the V-notched shear test. Franco and Gonzales [14] used the Vnotched test to investigate the shear strength of henequen fibre reinforced polyethylene. It was found that the shear strength of the composites ranges from $14 \mathrm{MPa}$ to $19 \mathrm{MPa}$. Almeida Jr et al. [8] investigated the shear strength of glass fiber reinforced epoxy composite using the same method and found that the shear strength was approximately $22.3 \mathrm{MPa}$ for $90 \mathrm{~s}$ fiber configuration and 70.4 MPa for a mat configuration. Almeida Jr et al. [15] also studied the effect of hybridization the mechanical properties of composite made of curaua/glass fiber reinforced polyester using the v-notched shear test. It was concluded that the hybridization enhanced the mechanical properties of the resulted composite, where the shear stress achieved was around 20-24 MPa. Fajrin [16] employed the same method to evaluate the shear stress of sisal/epoxy composite and it was found that the shear stress of the resulted composites ranged from $12 \mathrm{MPa}$ to $15 \mathrm{MPa}$. The aim of this work was to investigate the shear properties of two different types of natural fibers, jute and hemp fibers, when used to reinforce epoxy resin polymer. The obtained results were compared to the results of previous reported studies. The shear properties of those composites were provided using the V-notched or Iosipescu method.

\section{Materials and method}

\subsection{Sample preparation}

Two different types of natural fibres were used in this study, that is, jute and hemp fibers. The Jute fibres were provided in woven configurations, while hemp fibres were in the form of a hemp mat that obtained from an overseas supplier (China). A modified low viscosity epoxy resin (R180) with a hardener (H180) was used as the matrix with a composition ratio of 100:20 by weight. The jute and hemp fibres were chemically treated using sodium hydroxide $(\mathrm{NaOH})$ with a composition of $2 \%$ by weight $(2 \% \mathrm{wt})$. The fibers were also washed with warm tap water and dried at room temperature for 12 hours, as pre-treatment. The 
chemical treatment was conducted by soaking the natural fibres in a $2 \% \mathrm{NaOH}$, purchased with the commercial name of Formosoda-P at ambient temperature for 4 hours.

The treated fibres were then washed several times with warm tap water, neutralized with acetic acid and washed with demineralized water as a post-treatment. The fibres were allowed to dry for 3 days at room temperature. The samples were prepared using a vacuum bagging process. The resulted composite laminates were labeled as JFC and HFC for jute and hemp, respectively. The laminates are categorized as a transverse isotropic material since their properties are similar in the parallel and transverse direction.

\subsection{Testing method}

The Iosipescu or V-notched uses a rectangular beam that is loaded by a special fixture, applying a shear loading at the v-notch. The sample is inserted into the fixture with the notch located along the line of action of loading by means of an alignment that references the fixture. The testing machine compressed the two halves of the fixture while the load is observed. In this test, five specimens provided from each laminate were tested. The testing was conducted using an MTS machine equipped with a $10 \mathrm{kN}$ load cell. The testing machine was displacement controlled and set-up to apply a load with the speed of $2 \mathrm{~mm} / \mathrm{min}$. The test, as shown in Figure 1 (A), was conducted until the fracture occurs within the tested specimen. Figure 1 (B) shows the detail of the V-notched shear test configuration. According to the reference standard, ASTM D 5379M [13], the shear strength is the shear stress carried by a material at failure under a pure shear condition. The average shear stress is determined by dividing the applied load by the area of the cross-section between the notches.

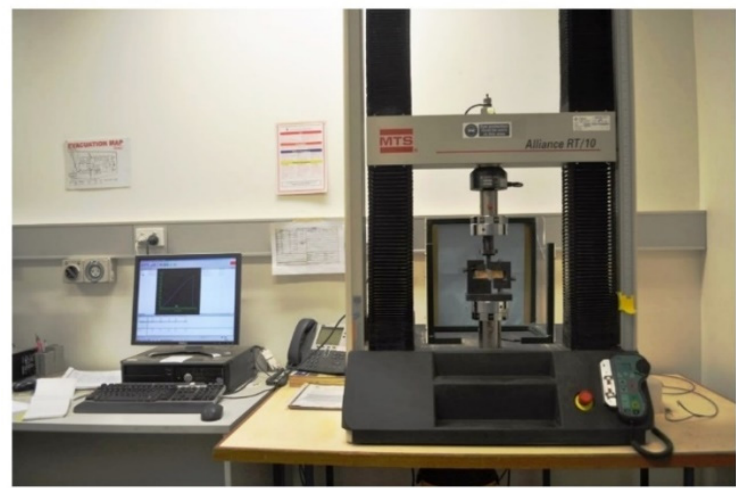

(A)

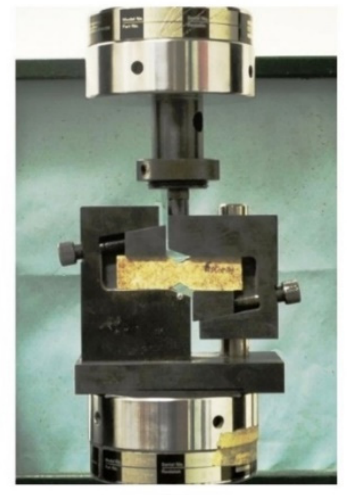

(B)

Fig. 1. Setting-up for the v-notched shear test.

\section{Results and discussions}

\subsection{Shear stress}

The tests results are tabulated in Table 1, which shows the deflections and shear stresses for all the tested samples. The data were also presented in the form of dot-plot and bar chart diagram in order to obtain more apparent information, as shown in Figure 2 (A) and Figure 2(B), respectively. The average shear stress of JFC is $25.57 \mathrm{MPa}$, which is $13.8 \%$ higher than the average shear stress of HFC, $22.46 \mathrm{MPa}$. It is visually shown in Figure 2 (A), that the data spread is disproportionate, particularly for the data of JFC. The shear stress of JFC ranges from 17.03 $\mathrm{MPa}$ to $29.83 \mathrm{MPa}$, with a coefficient of ratio (CV) of 14.46. The data of 
HFC has a much better distribution with a CV value of 11.75. Both are, however, within the acceptable limit of CV values for experimental data, which is according to Patel et al. [17], $33 \%$ for the upper limit of CV value. It is also clearly shown in the dot plot diagram that each data group has at least one or two outliers. The shear stress of sample 5 in JFC group should be considered as outliers as the value was approximately $75.16 \%$ lower than the highest value $(29.83 \mathrm{MPa})$ or $50.14 \%$ lower than the average value of the JFC group. Likewise, the shear stress of sample 4 within HFC group should also be considered as an outlier as it has the lowest value, $18.51 \mathrm{MPa}$, which is $38.78 \%$ lower than the highest in its group. The shear stress distribution of HFC appears relatively consistent, as seen in Figure 2 (A), although it has lower average values as shown in Figure 2 (B). In order to provide more reliable data, it is strongly recommended to do a normalization of the data by omitting the outliers.

Table 1. Shear properties of jute fiber composite (JFC) and hemp fibre composite (HFC).

\begin{tabular}{|c|c|c|c|c|}
\hline \multirow{3}{*}{ Specimen } & \multicolumn{2}{|c|}{$\begin{array}{c}\text { Jute Fiber Composite } \\
\text { (JFC) }\end{array}$} & \multicolumn{2}{c|}{$\begin{array}{c}\text { Hemp Fiber Composite } \\
\text { (HFC) }\end{array}$} \\
\cline { 2 - 5 } & $\begin{array}{c}\text { Deflection at } \\
\text { Peak (mm) }\end{array}$ & $\begin{array}{c}\text { Peak Shear } \\
\text { Stress (MPa) }\end{array}$ & $\begin{array}{c}\text { Deflection at } \\
\text { Peak (mm) }\end{array}$ & $\begin{array}{c}\text { Peak Shear } \\
\text { Stress (MPa) }\end{array}$ \\
\hline 1 & 1.71 & 29.83 & 2.08 & 22.94 \\
\hline 2 & 1.64 & 28.90 & 2.25 & 25.69 \\
\hline 3 & 1.99 & 29.68 & 1.98 & 23.48 \\
\hline 4 & 1.31 & 22.39 & 1.66 & 18.51 \\
\hline 5 & 1.66 & 17.03 & 1.89 & 21.69 \\
\hline Mean & 1.66 & 25.57 & 1.97 & 22.46 \\
\hline CV & 14.46 & 22.21 & 11.17 & 11.75 \\
\hline
\end{tabular}

The data obtained in this study were closely approaching other previous studies. As previously mentioned, Franco and Gonzales [14] found that composites made of henequen fibre reinforced polyethylene has a shear stress of $14 \mathrm{MPa}$ to $19 \mathrm{MPa}$. Almeida Jr et al. [8] provided a composite with a value of average shear stress around $22.3 \mathrm{MPa}$, which is very close to the shear stress of HFC in this study. In addition, a pretty similar average value was given by the work of Almeida Jr et al. [15] which was approximately 20-24 MPa. Further, a composite that is made of sisal/epoxy has a slightly lower shear stress, which was about 12 $\mathrm{MPa}$ to $15 \mathrm{MPa}$, and reported by Fajrin [16].

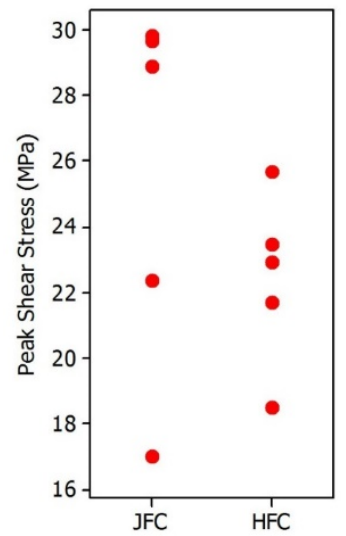

(A)

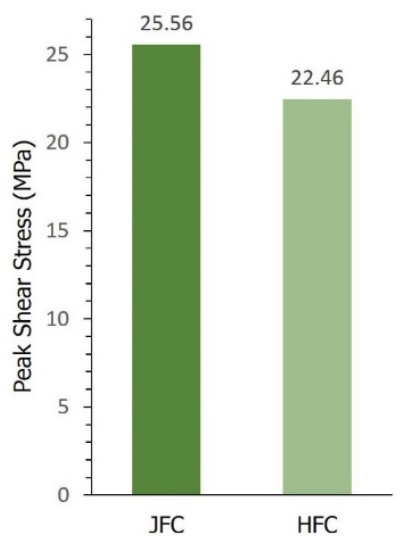

(B)

Fig. 2. The dot-plot diagram (A) and bar-chart diagram (B) of the testing results. 


\subsection{Load-displacement behaviour}

Figure 3(A) shows typical load-displacement curves of hemp fiber composite (HFC). A quick look at the graph gives an indication that the HFC laminate was properly and well prepared. All the curves for the 5 samples showed a nearly linear trend at the initial stage of loading and a slight deflection prior to reaching the maximum load. Four samples collapsed in an abrupt mode, which is a common failure mechanism of brittle material such as natural fiber composite. One sample, however, behaved just like a non-linear material, revealed a few steps after reaching the maximum value, which is indicating a delamination process within the sample. This particular behaviour was also reported by Almeida Jr et al. [8]. The typical load-displacement curves of jute fiber composite (JFC) is depicted in Figure 3 (B). As clearly shown in that figure, the load-displacement curves show a linear elastic behaviour at the initial stage, and after some point a reduction of the material stiffness is shown until the final failure. The graph also shows that three out of five specimens failed in a sudden failure mode, while two specimens demonstrated non-linear behaviour after reaching the maximum load.

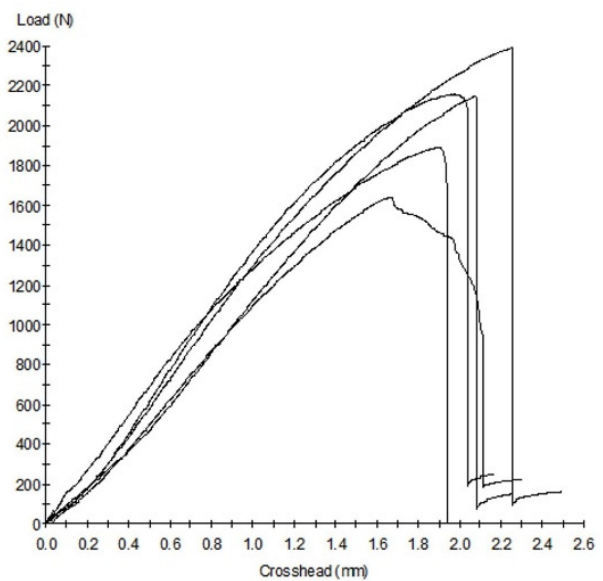

(A)

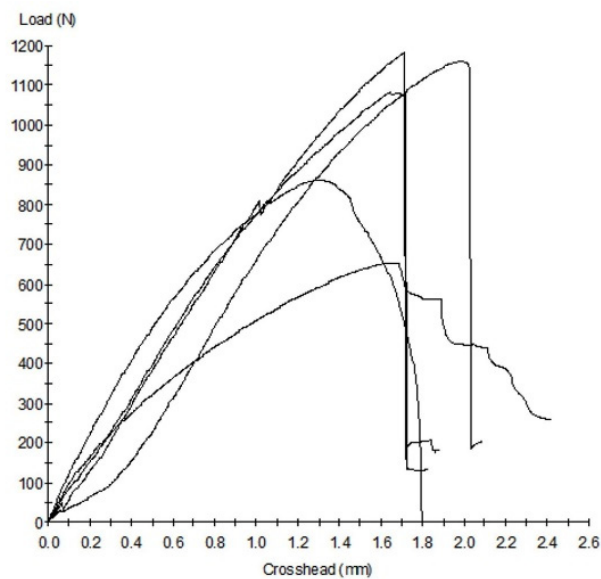

(B)

Fig. 3. Load-displacement graphs of hemp fiber composite (A) and jute fiber composite (B) in shear.

\subsection{Failure modes}

Figure 4 (A) shows a typical failure pattern of HFC specimens. Four out of five specimens were fractured in diagonal cracking, which also known as an off-axial failure mode, as shown in the figure. Only one single specimen actually failed across the notched section, which is strongly desired in this type of shear test. This specimen may be associated with a graph shown in Figure 3(A), showing a non-linear behaviour beyond the maximum load. Similarly, the failure mode of JFC specimens was also dominated by diagonal cracking, as shown in Figure 4(B). It was detected that the crack initiated at the upper or lower notched surfaces. The crack then developed in the direction close to $45^{\circ}$ from the principal axis. A similar failure mode was also reported by Yuan et al. [12]. Further, Yuan et al. [12] explained that a uniform or pure shear stress is generated in the center of the notched section when the specimen is under shear loading during the test. The shear stress distribution is parallel to the loading path which is in a vertical direction. At the same time, the principal stresses in compression and tension are also generated to the gage length of the sample in the direction of $\pm 45^{\circ}$. Furthermore, Archila et al. [18] explained that the pure shear stress between the notched is propagating by the action of two applied loads of equivalent magnitude subjected to the tested specimen. 


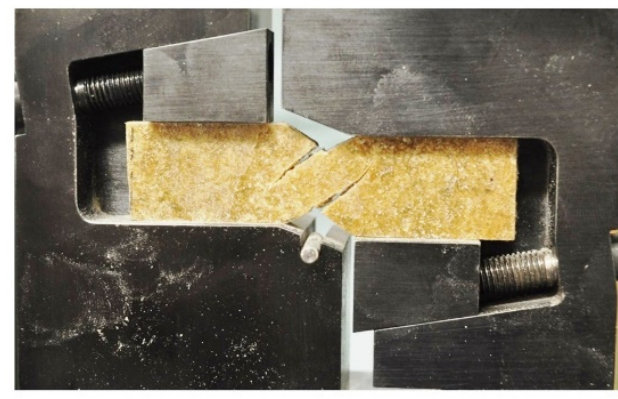

(A)

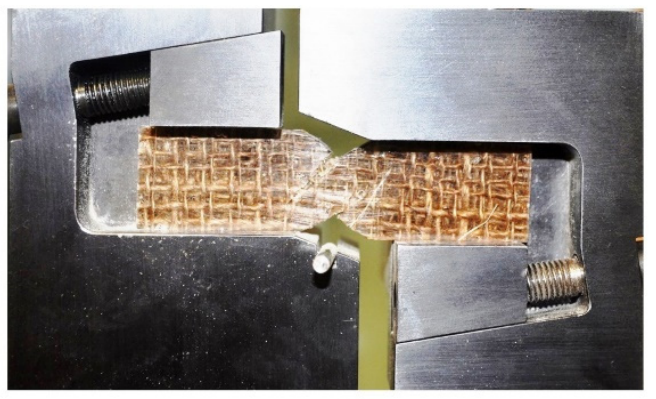

(B)

Fig. 4. Typical shear failure pattern of HFC specimen (A) and JFC specimen (B) under shear loading.

\section{Conclusions}

The current reported experimental study was focused on evaluating the shear properties of jute and hemp fiber composites using Iosipescu or the V-notched shear test. It was found that the jute fiber composites (JFC) have an average shear stress of $25.56 \mathrm{MPa}$, while hemp fiber composites (HFC) have an average value of $22.46 \mathrm{MPa}$. These values were very closely approaching the shear stress of other natural fiber composites tested using the same method. Regarding the load-displacement behaviour, both JFC and HFC specimens showed a nearly linear trend at the initial stage of loading that slightly deflected prior to reaching a maximum load. The majority of the specimens in both groups, JFC and HFC, were fractured in a diagonal cracking, which is also known as an off-axial failure mode. Only a limited number of specimens collapsed across the notched section. Both these failure modes are acceptable under this type of shear test.

\section{References}

1. B.C. Suddel, A. Rosemaund, Proceedings of the symposium on natural fibres, Industrial fibres: recent and current developments, Rome, (2008).

2. N.H. Sari, I.N.G. Wardana, Y.S. Irawan, E. Siswanto, Advances in Acoustics and Vibrations, Corn husk fiber -polyester composites as sound absorber: non acoustical and acoustical properties, 4, 1-8, (2017).

3. J. Fajrin, Civil Engineering Dimension, The application of statistical design of experiments to study the in-plane shear behaviour of hybrid composite sandwich panel, 18(1), 25-30, (2016).

4. J. Fajrin, Y. Zhuge, F. Bullen, H. Wang, Journal of Mechanical Engineering and Science, Flexural behaviour of hybrid sandwich panel with natural fiber composites as the intermediate layer, 10(2), 1968-1983, (2016).

5. J. Fajrin, Y. Zhuge, F. Bullen, H. Wang, Civil Engineering Dimension, Experimental and theoretical deflections of hybrid composite sandwich panel under four-point bending load, 19(1), 29-35, (2017).

6. J. Fajrin, Y. Zhuge, F. Bullen, H. Wang, International Journal of Technology, The structural behavior of hybrid structural insulated panels under pure bending load, 8(5), 777-788, (2017).

7. J. Fajrin, N.H. Sari, Jurnal Spektran, Tensile properties of lignocellulosic composites: A comparison analysis between natural fiber composites (NFCs) and medium density fiber (MDF), 6 (1), 111-116, (2018). 
8. J.H.S. Almeida Jr, C.C. Angrizani, E.C. Botelho, S.C. Amico, Materials and Design, Effect of fiber orientation on the shear behaviour of glass/epoxy composite, 65, 789795, (2015).

9. W.R. Broughton, Shear, in J.M. Hodgkinson, Mechanical testing of advanced fiber composites, CRC Press, Woodhead Publishing Ltd, (2000).

10. J. Fajrin, Jurnal Ilmu dan Teknologi Kayu Tropis, Compressive properties of tropical natural fibers reinforced epoxy polymer composites, 14(2), 127-135, (2016).

11. J. He, M.Y.M. Chiang, D.L. Hunston, C.C. Han, Journal of Composite Materials, Application of the $V$-notch shear test for unidirectional hybrid composites, 36, 26532666, (2002).

12. Y. Yuan, C. Feng, Z. Xu, H.P. Li, Experimental and Applied Mechanics, Investigation in shear behaviour of intermetallic composite using V-notched beam test method, 6, 5360, (2011).

13. ASTM Standard D 5379 M, Standard test method for shear properties of composite materials by the V-Notched beam method, ASTM D 5379 M-05, ASTM International, Philadelphia, Pa 19103, (2005).

14. P.J.H. Franco P.J., A.V. Gonzalez, Composites: Part B, A study of the mechanical properties of short natural-fiber reinforced composites, 36, 597-608, (2005).

15. J.H.S. Almeida Jr, S.C.Amico, E.C. Botelho, F.D.R. Amado, Composites: Part B, Hybridization effect on mechanical properties of curaua/glass fiber composites, $\mathbf{5 5}$, 492-497, (2013).

16. J. Fajrin, Info Teknik, Mechanical properties of natural fiber composite made of Indonesian grown sisal, 17(1), 69-84, (2016).

17. J.K. Patel, N.M. Patel, R.L. Shiyani, Current Science, Coefficient of variation in field experiments and yardstick thereof - An empirical study, 81(9), 1163-1164, (2001).

18. H.F. Archila, M.P. Ansell, P. Walker, 10th Wood Bamboo Congress, Korea, (2015). 